\title{
Turbodrills design and performance analysis for efficient drilling in hard rocks
}

\author{
A. Mokaramian ${ }^{1}$, V. Rasouli ${ }^{1}$ \& G. Cavanough ${ }^{2}$ \\ ${ }^{I}$ Deep Exploration Technologies Cooperative Research Centre \\ (DET CRC) and Department of Petroleum Engineering, \\ Curtin University, Australia \\ ${ }^{2}$ Deep Exploration Technologies Cooperative Research Centre \\ (DET CRC), and CSIRO, Queensland Centre for Advanced Technologies \\ (QCAT), Australia
}

\begin{abstract}
The authors have recently proposed Coiled Tube Turbodrilling technology for drilling deep hard rocks mineral exploration. Coiled Tube (CT) is a continuous length of ductile steel or composite tube that itself cannot rotate and therefore a down hole motor is needed to provide mechanical power to the bit. Amongst the down hole motors, turbodrills (turbine down hole motors) are an excellent fit with CT operations for hard rocks, providing a smooth borehole with little vibrational effects during drilling with high output rotational speed. The turbine motor section has multistage of rotors and stators which convert the hydraulic power to mechanical power.

This paper presents a methodology for designing turbodrills with asymmetric rotor or stator blades configurations. Here, the turbodrill is designed specifically for small size CT system providing suitable output power and rotation speed with applicable input flow properties. Also, the results of a few numerical simulations carried out using computational fluid dynamics (CFD) code are presented. The results help in choosing the best turbine motor configurations to obtain optimum rotational speed and torque during drilling hard rocks for small hole size exploration applications. Similar methodology can be used to design and choose the best turbodrill for other hard rocks drilling conditions.

Keywords: hydraulic turbodrill, turbine down hole motor, hard rocks, Coiled Tubing (CT) drilling, drilling efficiency, numerical simulation.
\end{abstract}




\section{Introduction}

Coiled Tube (CT) Turbodrilling technology has been recently proposed for drilling deep hard rocks for mineral exploration applications (Mokaramian et al. [1]). Coiled Tube (CT) is a continuous length of ductile steel or carbon fiber tube that is stored and transported over a large reel (see Fig. 1). In exploration applications, drilling small size holes as fast as possible and obtaining reliable samples to the surface yields several advantages over conventional drilling methods. Coiled tube (CT) allows fast drilling by eliminating the connection time and providing continuous circulation during drilling. This enables quick access to the zone of interest to collect the cuttings or obtain core samples.

Coiled tube itself cannot rotate and therefore a down hole motor is needed to provide mechanical power and rotation to the bit. There are many special design criteria to be considered for successful operation of down hole motors in CT drilling $[2,3]$. One major concern is that it is often difficult to produce enough weight on bit (WOB) to maximize the rate of penetration (ROP) for optimized drilling. Since the ROP of a fixed cutter drill bit is a product of the depth of cut (DOC) and the rotation speed and because the DOC is primarily produced by the available WOB, in an environment where WOB is limited (as with CT drilling); high rotation speed is the key driver for ROP (Beaton and Seale [2]). Amongst available down hole motors, turbodrills (turbine motors) are the best choice to be used for small size CT drilling hard rocks (Mokaramian et al. [4]): this results in a smooth borehole with little vibrational effects during drilling and produce a high quality hole. The turbine motor section has multistage of stators and rotors which convert the hydraulic power provided by the drilling fluid to mechanical power.

In general, the down hole turbine motor (Turbodrill) is composed of two sections: turbine motor section and bearing section i.e. thrust-bearing and radial support bearing (Eskin and Maurer [5]). Figure 2 shows a typical Turbodrill assembly and the fluid flow path through turbine stages.

The activating drilling fluid is pumped at high velocity through the motor section, which, because of the vane angle of each rotor and stator, causes the rotor to rotate the shaft of the motor which is connected to the bit. The energy required to change the rotational direction of the drilling fluid is transformed into rotational and axial (thrust) force. This energy transfer is seen as a pressure drop in the drilling fluid. In practice, multiple stages are stacked coaxially until the desired power and torque is achieved.

A turbine device has the unique characteristic that it will allow mud circulation independent of motor's torque or power. If the turbine motor is lifted off the bottom of the borehole while mud circulation continues, the motor will speed up to the runaway speed and the motor produces no drilling torque or power. As the turbine motor is lowered and weight is placed on the motor and thus the bit, the motor begins to slow its speed and produce torque and power. When sufficient weight is applied on the turbine motor, the motor will produce its maximum possible power. If more weight is added to the turbine motor and the bit, the motor speed and power output will continue to decrease and torque 
continuously will increase till the motor cease to rotate and the motor is described as being stalled (Lyons and Plisga [6]). At this condition, the turbine motor produces its maximum possible torque. Even when the motor is stalled, the drilling fluid is still circulating.

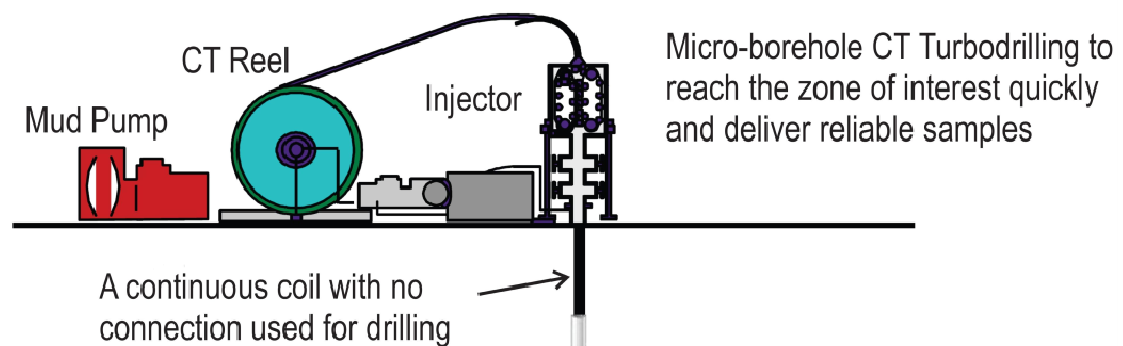

A downhole turbine motor is used to rotate the bit

Impregnated bit must be used for drilling in hard and abrasive rocks
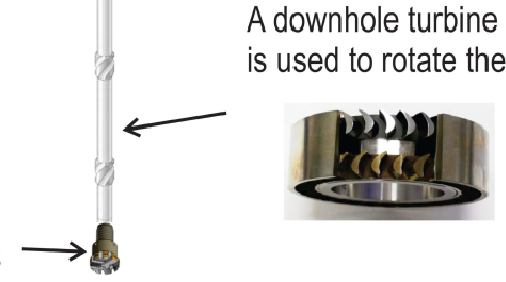

Figure 1: Schematic of the coiled tubing unit for hard rocks exploration drilling.

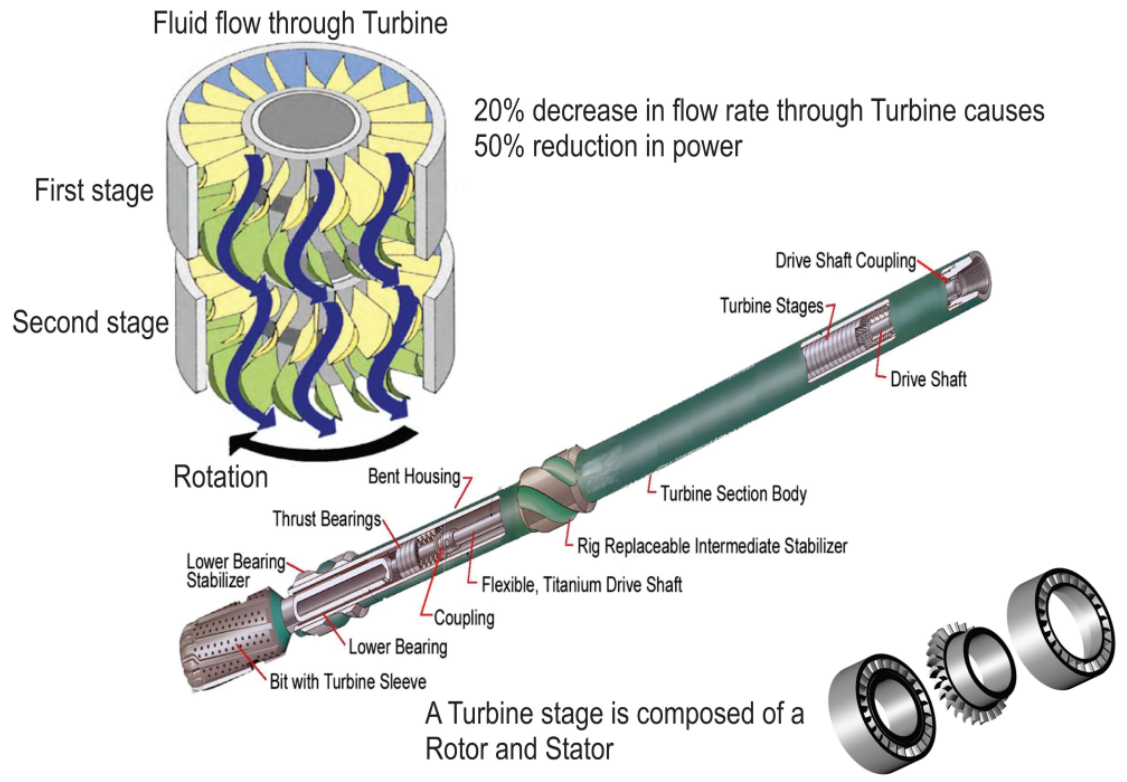

Figure 2: Turbodrill assembly and schematic fluid flow through turbine stages, after Beaton and Seale [2]. 
It is almost impossible to determine formulae for turbines under working conditions (Eskin and Maurer [5]). Laboratory experiments using drilling dynamometer test stand have to be conducted to determine nominal data on Turbodrills. If the performance parameters for a turbine motor design are known for a given circulation flow rate and mud weight, the performance parameters for other circulation flow rate and mud weight can be found using the following mathematical relationships (Eskin and Maurer [5]):

$$
\begin{aligned}
& N \propto Q ; T \propto Q^{2} ; \Delta p \propto Q^{2} ; P \propto Q^{3} ; \eta \propto \frac{1}{Q}, \\
& N \propto \frac{1}{\rho} ; T \propto \rho ; \Delta p \propto \rho ; P \propto \rho ; \eta \propto \frac{1}{\rho},
\end{aligned}
$$

where:

$N=$ Turbodrill output rotation speed (revolution per minute),

$\rho=$ Drilling fluid (mud) density,

$P=$ Turbodrill output power,

$T=$ Turbodrill output Torque,

$Q=$ Flow rate,

$\Delta p=$ Turbodrill differential pressure,

$\eta=$ Turbodrill efficiency.

Depending on the skills of the turbodrill operator the speed may vary from zero rpm to the runaway speed. The turbine motor instantaneous torque $T$ (N.m) for any rotation speed $N(\mathrm{rpm})$ is:

$$
T=T_{s}\left(1-\frac{N}{N_{r}}\right),
$$

where:

$T_{s}=$ Stalled torque (maximum torque),

$N_{r}=$ Runaway rotation speed (maximum speed).

Many new turbodrill designs and modifications are currently underway to extend the applicability of Turbodrilling to coiled tubing operations (Radtke et al. [7], Grigor et al. [8], Seale et al. [9]). One of the most significant developments in progress is the creation of a turbodrill that is much shorter than existing designs in order to enhance compatibility with coiled tube equipment.

\section{Turbodrill design}

When designing a hydraulic multistage turbine, it is assumed that each turbine stage is identical and that the flow rate, pressure drop, rotary speed, generated torque, and power transmitted to the shaft are the same for each of the stages. The simplest approach for turbine analysis is to assume that the flow conditions at a mean radius, called the pitchline, represent the flow at all radii (Dixon and Hall [10]). The well-known method of building velocity triangles is used when designing the blade unit profile. Fluid enters the stator with an absolute velocity $c_{1}$ and at an absolute velocity angle $\alpha_{1}$ and accelerates to an absolute velocity $c_{2}$ at angle $\alpha_{2}$. From the velocity diagram, the rotor inlet relative velocity $w_{2}$, at a relative velocity angle $\beta_{2}$, is found by subtracting, vectorially, the blade speed, $U$, 
from the absolute velocity $c_{2}$. The relative flow within the rotor accelerates to velocity $w_{3}$ at an angle $\beta_{3}$ at rotor outlet.

Three key non-dimensional parameters are related to the shape of the turbine velocity triangles and are used in fixing the preliminary design of a turbine stage. These are described in the following sections.

\subsection{Design flow coefficient}

Design flow coefficient is defined as the ratio of the meridional flow velocity to the blade speed, $\varnothing=c_{m} / U$ (Dixon and Hall [10]). For turbodrill as an axial turbomachinery, to simplify the analysis it is usually assumed that the flow does not vary in the tangential direction (i.e. $c_{m}=c_{x}$ ). Therefore the flow coefficient for turbodrill is defined as:

$$
\phi=\frac{c_{x}}{U} .
$$

The value of $\varnothing$ for a stage determines the relative flow angles. A stage with a low value of $\varnothing$ implies highly staggered blades and relative flow angles close to tangential axis, whereas high values imply low stagger and flow angles closer to the axial axis (Dixon and Hall [10]).

\subsection{Stage loading coefficient}

The stage loading is defined as the ratio of the stagnation enthalpy change through a turbine stage to the square of the blade speed, $\psi=\Delta h_{0} / U^{2}$. In turbodrill that is assumed to be an adiabatic turbine, the stagnation enthalpy change is equal to the specific work, $\Delta \mathrm{W}$, and because it is a purely axial turbine with constant radius, we can use the Euler work equation $\left(\Delta \mathrm{W}=U \times \Delta c_{\theta}\right)$ to write, $\Delta h_{0}=U \times \Delta c_{\theta}$ (Dixon and Hall [10]). As a result, the stage loading for turbodrill can be written as:

$$
\psi=\frac{\Delta c_{\theta}}{U},
$$

where $\Delta c_{\theta}$ represents the change in the tangential component of absolute velocity through the rotor. Thus, high stage loading means large flow turning and leads to highly "skewed" velocity triangles to achieve this turning. Since the stage loading is a non-dimensional measure of the work extraction per stage, a high stage loading is desirable because it means fewer stages needed to produce a required work output (Dixon and Hall [10]). As a result, with designing blades leading to large flow turning, the stage loading coefficient will be higher and smaller turbodrill with lesser stages can result to the required power as long as being limited by the effects that high stage loadings have on efficiency.

\subsection{Stage reaction}

The stage reaction is defined as the ratio of the static enthalpy drop in the rotor to the static enthalpy drop across the turbine stage. Taking the flow through a turbodrill as isentropic, the equation of the second law of thermodynamics, 
$T d s=d h-d p / \rho$ can be approximated by $d h=d p / \rho$, and ignoring compressibility effects, the reaction can thus be obtained as (Dixon and Hall [10]):

$$
R=\frac{p_{2}-p_{3}}{p_{1}-p_{3}} \text {. }
$$

The reaction therefore indicates the drop in pressure across the rotor compared to that of the stage. It describes the asymmetry of the velocity triangles and is therefore a statement of the blade geometries (Dixon and Hall [10]). For example, a $50 \%$ reaction turbodrill implies velocity triangles that are symmetrical, which leads to similar stator and rotor blade shapes. Typically, in prior turbodrills design a 50\% reaction was selected (i.e. the stator blades and the rotor blades are symmetric) (Natanael et al. [11]).

The main goal in the preliminary stage design of a turbodrill is to fix the shapes of the velocity triangles, either by setting the flow angles or by choosing values for the three dimensionless design parameters, $\varnothing, \psi$, and $R$.

From the specification of the turbodrill, the design will usually have a known mass flow rate of the drilling fluid and a required power output. As a result, the specific work per stage can be determined from the stage loading and the blade speed and, consequently, the required number of stages can be found as follows:

$$
n_{\text {stage }} \geq \frac{\Delta W}{\psi U^{2}}=\frac{P_{\text {hyd }}}{\dot{m} \psi U^{2}},
$$

where, $P_{\text {hyd }}$ is output turbodrill power and $\dot{m}$ is the fluid mass flow rate. An inequality is used in this equation, because the number of stages must be an integer value. The equation here shows how a large stage loading can reduce the number of stages required in a multistage turbodrill. Also it shows that a high blade speed, $U$, is desirable as it will reduce the number of required stages.

For turbodrills, several useful relationships can be derived relating the shapes of the velocity triangles to the three dimensionless design parameters. These relationships are important for the preliminary design. Starting with the definition of the stage reaction, and accepting no work is done through the stator, so the stagnation enthalpy remains constant across it and after proper substitutions, finally the relations between flow coefficients and angles are obtained as follows:

$$
R=\frac{\phi}{2}\left(\tan \beta_{3}-\tan \beta_{2}\right)=1-\frac{\phi}{2}\left(\tan \alpha_{2}-\tan \alpha_{1}\right) .
$$

Also it can be obtained that:

$$
\psi=2\left(1-R+\phi \tan \alpha_{1}\right)
$$

Two important angles in the geometry of a rotor vane are $\beta_{2}$ and $\beta_{3}$. These two angles are important factors in the performance of the rotor vane because they determine the change in the direction of the drilling fluid passing through the rotor blade. $\beta_{2}$ plus $\beta_{3}$ is preferably less than 120 degree to avoid excessive blade turning, which can damage the rotor vanes (Natanael et al. [11]). The work done on the rotor by unit mass of fluid, the specific work equals the stagnation enthalpy drop incurred by the fluid passing through the stage and according to the Euler work equation this can be written mathematically as: 


$$
\Delta W=\Delta h_{0}=U \Delta c_{\theta}=U c_{x}\left(\tan \beta_{2}+\tan \beta_{3}\right)
$$

The output power of each stage is obtained as follows:

$$
P=\rho Q \Delta W=\rho Q U c_{x}\left(\tan \beta_{2}+\tan \beta_{3}\right) .
$$

Also the output torque of each stage is:

$$
T=\rho Q r_{m} c_{x}\left(\tan \beta_{2}+\tan \beta_{3}\right) .
$$

With the profile of the stator and rotor vanes being defined, an optimum number of blades per stator and rotor and the chord lengths of the blades can also be estimated during the preliminary design. The aspect ratio of a blade row is the height, or blade span, divided by the axial chord, $H / b$. A suitable value of this is set by mechanical and manufacturing considerations and will vary between applications. Typically, in prior turbodrills design the aspect ratio for rotor blades was 0.5. It has been found that energy losses may be reduced by increasing the aspect ratio of the stator and/or rotor blades (Natanael et al. [11]). To find the ratio of blade pitch to axial chord, $S / b$, the Zweifel criterion for blade loading can be applied. The Zweifel criterion states that for turbine blades there is an optimum space-chord ratio that gives a minimum overall loss. Typically, the Zweifel criterion, $Z$, is assumed to be between 0.5 and 1.2 (Dixon and Hall [10]). If the spacing between the blades is made small, the fluid receives the maximum amount of guidance from the blades, but the friction losses will be large. On the other hand, with the same blades spaced well apart, friction losses are small but, because of poor fluid guidance, the losses resulting from flow separation are high (Dixon and Hall [10]).

For a known axial chord, knowing $S / b$ fixes the number of blades on each stator and rotor row as (Dixon and Hall [10]):

$$
N_{B}=\frac{4 \pi r_{m} \times \cos ^{2} \beta_{3}\left(\tan \beta_{2}+\tan \beta_{3}\right)}{Z \times b} .
$$

\section{Numerical simulations}

In this study with the objective of fluid flow simulations with Computational Fluid Dynamics (CFD) through small diameter turbodrill suitable for CT Turbodrilling system, ANSYS 14.0 TurboSystem tools together with ANSYS CFX were utilized to investigate the turbodrill performance with different mass flow rates, various fluid types and rotation speeds. Here, the objective is to increase optimized output power and rotation speed suitable for hard rocks CT Turbodrilling system to reach higher drilling rates.

The shroud (housing) and hub (shaft) diameters of the turbodrill for the applications of this study were set to be 5 and $2.6 \mathrm{~cm}$, respectively. Consequently, the spanwise height $(\mathrm{H})$ will be $1.2 \mathrm{~cm}$ for this model with no shroud tip between blade tip and housing, i.e. the blades are connected to the housing. The axial chord is $1.5 \mathrm{~cm}$ with 14 blades on both stator and rotor rows.

In overall, the simulation results show that the turbodrill performance is highly dependent on the mass flow rate of the drilling fluid. As the mass flow rate is increased the expected optimum rotation speed and output power of the 
turbodrill increases. While the mass flow rate of the drilling fluid reduces, the output rotation speed, the power and consequently the shaft torque will drastically decrease to allow the velocity vectors entering rotor be aligned with the rotor inlet angle (to be effective in producing rotational effects on the rotor blades). Here, only a few of the simulation results for water are reported. As the drilling fluids are getting complex (multiphase) in terms of rheological properties, the fluid flow simulations through turbodrill are very useful to calculate the motor pressure drop and generated power especially in UBD CT operations that maintaining bottom hole pressure in the safe margin are crucial. Figures 3 and 4 shows the CFD simulation results for water and with 6 and $7 \mathrm{Kg} / \mathrm{s}$ of mass flow rates respectively and rotation speed of 3,000 rpm.

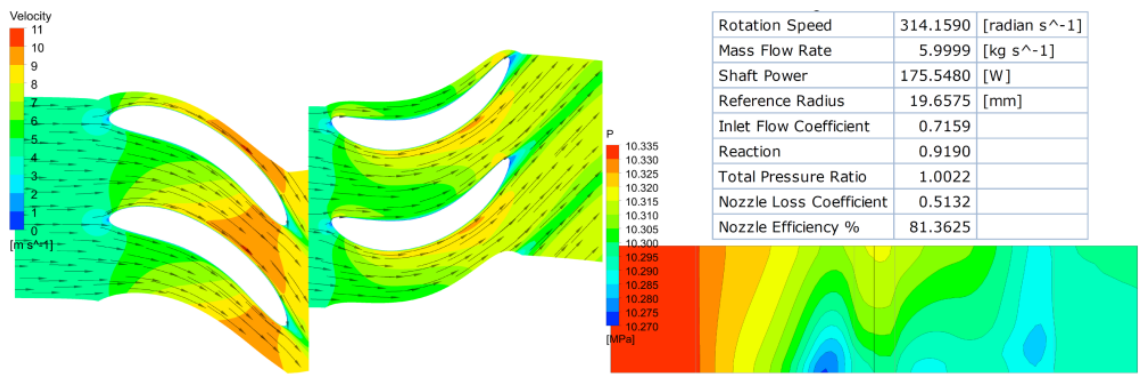

Figure 3: Water flow simulation results with mass flow rate of $6 \mathrm{Kg} / \mathrm{s}$ and rotation speed of 3,000 rpm.

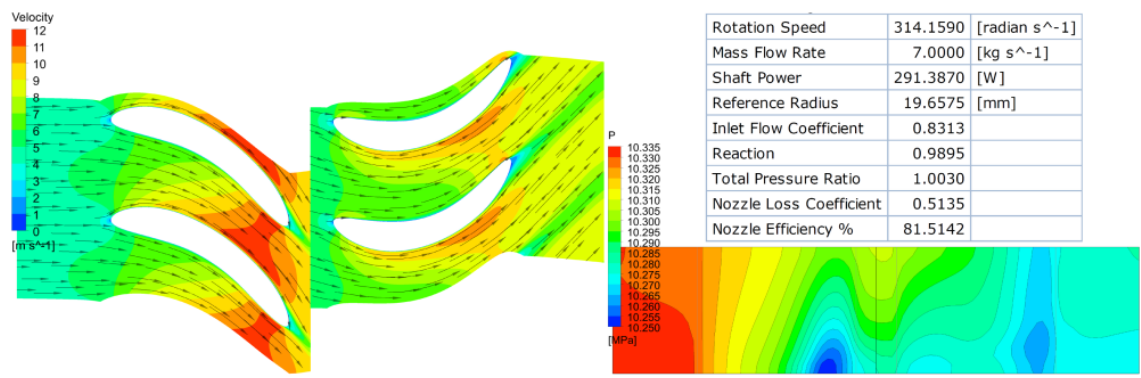

Figure 4: Water flow simulation results with mass flow rate of $7 \mathrm{Kg} / \mathrm{s}$ and rotation speed of 3,000 rpm.

Figure 5 shows the output power and torque trends from the simulation results versus rotation speed (rpm) for the two water mass flow rates of 6 and $7 \mathrm{Kg} / \mathrm{s}$. The simulation results show a very good match for the proposed turbodrill model. With this chart, the resulted power and torque in any other rotation speeds can be obtained with a very good accuracy. For water, due to limitations on the mass flow rates that can be injected from the surface pump down through the $\mathrm{CT}$ assembly to bottom hole to actuate the turbodrill (having huge pressure 
losses through the drilling assembly), the practical mass flow rates for this small size CT assembly should be below $7 \mathrm{Kg} / \mathrm{s}$, and here with the mass flow rate of $7 \mathrm{Kg} / \mathrm{s}$, optimum rotation speed is below 4,000 rpm and is around 3,000 rpm.

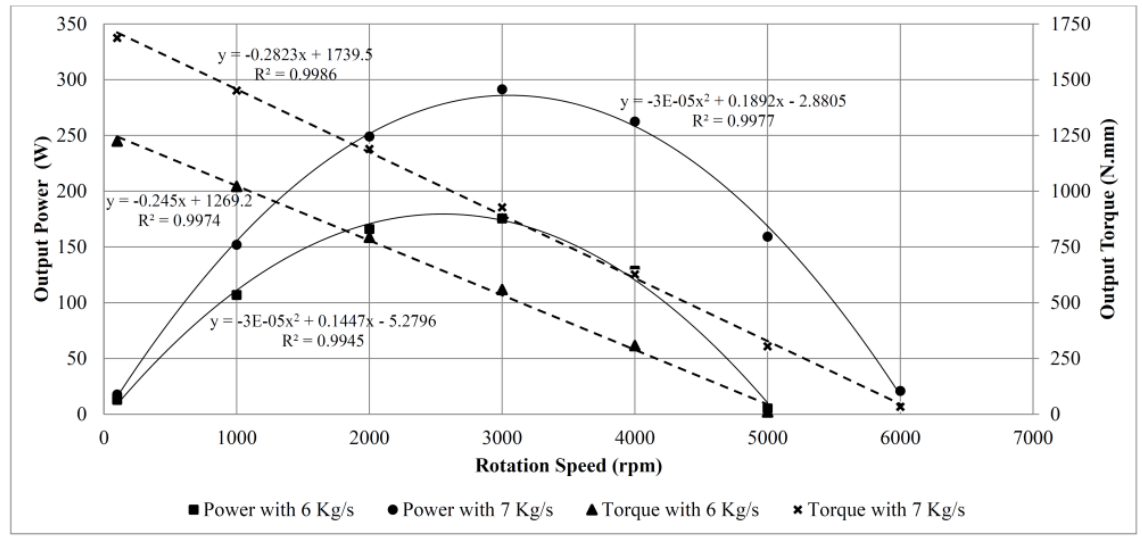

Figure 5: Water simulation results of one stage proposed turbodrill for the two mass flow rates of 6 and $7 \mathrm{Kg} / \mathrm{s}$ with different rotation speeds.

\section{Power for hard rocks drilling}

Efficient drilling of hard rocks requires a comprehensive knowledge of the energy spent at the bit. The bit-rock interface and interaction laws determine the amount of the mechanical power that an impregnated diamond bit requires for efficient drilling and delivering the desired range of ROP. In a model of the drilling response for drag bits, a set of relations between the WOB $(W)$, the torque-on-bit, $(T)$, velocity ratio $V / \Omega$. These three basic variables are defined as, [12]:

$$
\begin{gathered}
w=\frac{W}{a}, \\
t=\frac{2 T}{a^{2}}, \\
d=\frac{2 \pi V}{\Omega},
\end{gathered}
$$

where $a$ is the bit radius. Scaling of the weight and torque removes the influence of the bit dimension from the interface laws. The scaled quantities $w$ and $t$, which have dimensions of force/length (a convenient unit is the N.mm ${ }^{-1}$ ), can conveniently be interpreted as the normal and shear force per unit length on a two-dimensional cutter removing material over a DOC $(d)$.

For full bit face CT drilling in hard rocks, it is preferred to design the drilling system for ultra-high rotation speeds (i.e. $N>2,000 \mathrm{rpm}$ ) and very low DOCs in 
the range of $0.01-0.1 \mathrm{~mm} / \mathrm{rev}$. For calculation purposes we assume $\mathrm{ROP} \approx 10 \mathrm{~m} / \mathrm{hr}$ at a rotation speed of $N \approx 3,000 \mathrm{rpm}(314.16 \mathrm{rad} / \mathrm{s})$. This results in a DOC of:

$$
d\left(\frac{\mathrm{mm}}{\mathrm{rev}}\right)=\frac{\operatorname{ROP}\left(\frac{\mathrm{m}}{\mathrm{hr}}\right) \times 16.7}{N(\mathrm{rpm})}=\frac{10 \times 16.7}{3000}=0.056 \frac{\mathrm{mm}}{\mathrm{rev}}
$$

To be on the conservative side, for hard rocks (UCS $\geq 100 \mathrm{MPa}$ ), the range of parameters mentioned above, the scaled weight and torque is considered to be:

$$
w \approx 200\left(\frac{N}{\mathrm{~mm}}\right) ; t \approx 50\left(\frac{\mathrm{N}}{\mathrm{mm}}\right)
$$

Here, the bit radius $(a)$ assumed to be $35 \mathrm{~mm}$ and therefore WOB $(W)$ and torque $(T)$ are calculated as:

$$
\begin{gathered}
w=\frac{W}{a} \Rightarrow W O B=200 \times 35=7 \mathrm{KN} . \\
t=\frac{2 T}{a^{2}} \Rightarrow T=\frac{50 \times(35)^{2}}{2}=30.6 \mathrm{~N} . \mathrm{m} .
\end{gathered}
$$

We have assumed here that the cutting processes are not intrinsically affected by down hole condition in particular the down hole mud pressure. The angular power $P$ at the bit required to drill is simply given by:

$$
P=T \Omega,
$$

where, $\Omega$ is the bit angular velocity (expressed in $\mathrm{rad}^{-\mathrm{s}^{-1}}$ ). This equation can be written in the following form:

$$
P_{m}(K W)=\frac{T(N . m) \times N(\mathrm{rpm})}{9,550},
$$

If the rotation speed is assumed to be $N \cong 3,000(\mathrm{rpm})$, then:

$$
P_{m}=\frac{30.6 \times 3,000}{9,550}=9.6 \mathrm{KW}(12.9 \mathrm{hp})
$$

The down hole motor output power should be adequate to provide the required power by the bit. This is to say that:

$$
P_{\text {hyd }} \geq P_{m},
$$

The hydraulic power generated by turbodrill $\left(P_{h y d}\right)$ is calculated as:

$$
P_{h y d}(h p)=\frac{Q(g p m) \times \Delta P(p s i)}{1714} \times \eta_{m},
$$

where $\eta_{m}$ is the Turbodrill efficiency, which is usually considered 0.5-0.6 (Eskin and Maurer [5]). Here, we use $\eta_{m}=0.6$, and the mud flow rate for the current application is considered to be $Q=95 \mathrm{gpm}(6 \mathrm{~L} / \mathrm{s})$. This would lead to the Turbodrill pressure drop $(\Delta P)$ of:

$$
12.9 \mathrm{hp}=\frac{95 \times \Delta P}{1714} \times 0.6 \Rightarrow \Delta P=388 \text { psi }(26.8 \mathrm{bar})
$$

For the turbodrill model used in this paper, according to the reference radius and the angular velocity it can be obtained as: 


$$
U=\omega \times r=314.159(\mathrm{rad} / \mathrm{s}) \times 0.0196575(\mathrm{~m})=6.17 \mathrm{~m} / \mathrm{s} .
$$

Using the simulation results for calculating $\Delta c_{\theta}$ and putting in eqn (5) the stage loading coefficient can be calculated for the two mass flow rates as follows:

$$
\begin{gathered}
\dot{m}=6(\mathrm{Kg} / \mathrm{s}) \Rightarrow \Delta c_{\theta}=4.8 \mathrm{~m} / \mathrm{s} \Rightarrow \psi=\frac{4.8}{6.17}=0.78 \\
\dot{m}=7(\mathrm{Kg} / \mathrm{s}) \Rightarrow \Delta c_{\theta}=6.8 \mathrm{~m} / \mathrm{s} \Rightarrow \psi=\frac{6.8}{6.17}=1.1 .
\end{gathered}
$$

Consequently, the number of stages required can be obtained from eqn (7) for the two mass flow rates of 6 and $7 \mathrm{Kg} / \mathrm{s}$ with 3,000 rpm as follows:

$$
\begin{gathered}
\dot{m}=6(\mathrm{Kg} / \mathrm{s}) \Rightarrow n_{\text {stage }} \geq \frac{9600(\mathrm{~W})}{6 \times 0.78 \times(6.17)^{2}} \Rightarrow n_{\text {stage }} \geq 53.9 \Rightarrow n_{\text {stage }}=54 \\
\dot{m}=7(\mathrm{Kg} / \mathrm{s}) \Rightarrow n_{\text {stage }} \geq \frac{9600(\mathrm{~W})}{7 \times 1.1 \times(6.17)^{2}} \Rightarrow n_{\text {stage }} \geq 32.7 \Rightarrow n_{\text {stage }}=33 .
\end{gathered}
$$

Similarly, the number of required stage can be obtained with dividing the total required power to the resulted power of each stage from simulation results. As a result, the length of the turbine motor section can be obtained having the stage length. Here, the stage length is $4-5 \mathrm{~cm}$. Therefore the length of turbine motor section for application of this study for the water mass flow rates of 6 and $7 \mathrm{Kg} / \mathrm{s}$ will be around $2.7 \mathrm{~m}$ and $1.7 \mathrm{~m}$, respectively.

\section{Conclusions}

Basic design methodology for a small multistage Turbodrill (turbine down hole motor) optimised for small size Coiled Tube (CT) Turbodrilling system for deep hard rocks exploration applications was presented. The results of a few numerical simulations for turbodrill stage performance with different water mass flow rates and rotation speeds using the Computational Fluid Dynamics (CFD) code were reported. The simulation results included in this paper for the specific blades configuration show a very good match for the proposed turbodrill model and they showed that the turbodrill performance is highly dependent on the mass flow rate of the drilling fluid, i.e. as the mass flow rate increases the expected rotation speed of the turbodrill and consequently the output power and torque will increase. Also, for a given range of rotation speeds and drilling fluid flow rates, the turbodrill specifications (blade numbers on each row, inlet and outlet angles, etc.) should be designed specifically in a way that it achieves the desired mechanical power with a high efficiency.

The methodology for power requirements for hard rocks drilling using the bitrock interface and interaction laws was presented and using the CFD simulation results for one stage, other design parameters of turbodrill including number of stages and the length of the turbine motor section were resolved. The same approach will be applied using any types of drilling fluid (multiphase fluids), different flow rates and etc. to optimize turbodrill for the desired drilling conditions. 


\section{Acknowledgements}

The authors would like to express their sincere thanks to the Deep Exploration Technologies Cooperative Research Centre (DET CRC) for their financial supports towards this project. This paper is the DET CRC document 2012/063.

\section{References}

[1] Mokaramian A, Rasouli V, Cavanough G, A feasibility study on adopting coiled tubing drilling technology for deep hard rock mining exploration, Deep Mining 2012: Proceedings of the sixth International Seminar on Deep and High Stress Mining, pp. 487-99, Perth, Western Australia, 2012.

[2] Beaton T, Seale R, The Use of Turbodrills in Coiled Tubing Applications, SPE/ICoTA Coiled Tubing Conference (SPE 89434). Houston, Texas, U.S.A., 2004.

[3] IT, Advanced Ultra-High Speed Motor for Drilling, (Impact Technologies LLC). Tulsa, Oklahoma, Final Report, US Department of Energy, National Energy Technology Laboratory (NETL), p., 2007.

[4] Mokaramian A, Rasouli V, Cavanough G, Adapting oil and gas downhole motors for deep mineral exploration drilling, Deep Mining 2012: Proceedings of the sixth International Seminar on Deep and High Stress Mining, pp. 475-86, Perth, Western Australia, 2012.

[5] Eskin M, Maurer WC, Advanced Downhole Drilling Motors, Maurer Engineering Inc., 1997.

[6] Lyons WC, Plisga GJ, Standard Handbook of Petroleum \& Natural Gas Engineering, 2nd ed, United States of America, Elsevier Inc., 2005.

[7] Radtke R, Glowka D, Rai MM, Beaton T, Seale R, High-Power Turbodrill And Drill Bit For Drilling With Coiled Tubing, Technology International, Inc., US Department of Energy, National Energy Technology Laboratory (NETL), 2011.

[8] Grigor C, Conroy D, Henderson M, Expanding the Use of Turbodrills in Coiled Tubing and Workover Applications, SPE/ICoTA Coiled Tubing and Well Intervention Conference and Exhibition (SPE 113721). The Woodlands, Texas, USA, 2008.

[9] Seale R, Beaton T, Flint G, Optimizing Turbodrill Designs for Coiled Tubing Applications, SPE Eastern Regional Meeting (SPE 91453). Charleston, West Virginia, USA, , p., 2004.

[10] Dixon SL, Hall CA, Fluid Mechanics and Thermodynamics of Turbomachinery, Sixth ed, The United States of America, Elsevier Inc., 2010.

[11] Natanael M, Nevlud KM, Beaton T, Beylotte J, Turbodrill with asymetric stator and rotor vanes, US Patent U.S.A, Smith International, Inc., p., 2008.

[12] Detournay E, Richard T, Shepherd M, Drilling response of drag bits: Theory and experiment, international Journal of Rock Mechanics and Mining Science;Vol. 45, pp. 1347-60, 2008. 